\title{
GENOMICS
}

\section{Fighting fire with fire}

\author{
Daniel F. Voytas
}

\section{Mobile genetic elements called transposons could cause havoc in the genome if left unregulated. Of the various cellular defence strategies used to preserve genome integrity, one involves exploiting transposons themselves.}

The college sophomores in my genetics class become visibly uncomfortable during my lectures on transposable elements. "You mean to tell me there are things jumping around my genome?" they ask when I explain that much of their genetic material is made up of DNA segments that can move about their chromosomes. The mobility of transposable elements is understandably disconcerting, because it would be expected to lead to chromosome rearrangements and mutations that could cause disease. In the long term, however, these elements are probably beneficial, serving as a powerful force for genome change and evolution.

Although the long-term view may do little to appease a 20-year-old, my students can take some comfort in the findings of Cam et al. ${ }^{1}$ reported in this issue (page 431). The authors show that, in the fission yeast Schizosaccharomyces pombe, proteins called CENP-Bs are drafted in to quell transposon activity. What is unusual about this particular choice is that CENP-Bs themselves are derived from transposable elements.

CENP-Bs were first discovered because they bind to repetitive DNA sequences called centromeres ${ }^{2}$. During cell division, centromeres assemble proteins to serve as anchor points for the spindle fibres that move chromosomes to the daughter cells. Fission yeast has three closely related CENP-Bs that bind to centromeres and that are crucial for centromere function ${ }^{3}$.

Cam et al. wanted to know whether CENP-Bs also associate with genomic regions other than centromeres. They found that, indeed, CENP-Bs occur at dispersed DNA repeats, particularly at the Tf2 transposable elements that litter the $S$. pombe genome. The authors show that CENP-Bs bind to Tf 2 and recruit enzymes known as histone deacetylases, which turn off Tf 2 expression and prevent transposition. The silenced Tf 2 elements are then packaged into subdomains of the nucleus called Tf bodies (Fig. 1).

But transposition can on rare occasions be advantageous, particularly in times of stress, as it has the ability to create potentially beneficial genetic variants ${ }^{4}$. When Cam and colleagues exposed $S$. pombe cells to oxidative stress, Tf2 elements became unbundled from Tf bodies and were expressed (Fig. 1). Furthermore, in cells lacking certain CENP-Bs, the Tf2-related element Tf1 was mobilized, making new insertions that were then packaged into Tf bodies. These observations indicate that CENP-Bs regulate Tf activity through selective packaging.
The CENP-B proteins are an unexpected choice for combating mobile elements because they originate from transposase ${ }^{5}$, the enzyme necessary for cutting and pasting DNA during transposition. The transposase progenitor of CENP-Bs belongs to a class of mobile element that is unrelated to Tf2. The authors argue that this unrelatedness suggests a conflict between different classes of mobile element, but this view is probably unnecessarily provocative. As transposases normally bind to DNA, they are suited to being moulded over time into genome regulators. In the case of CENP-B, the vestigial DNA-binding domain of the transposase recognizes centromeric repeats and Tf 2 elements. So, whereas CENP-Bs can no longer cut and paste DNA, they can recruit histone deacetylases to regulate Tf2. Other transposasederived proteins also act as gene regulators. For example, two transcriptional activators used by plants in their response to light are derived from transposases ${ }^{6}$, and it is speculated that many such transposase-derived proteins serve crucial roles in gene regulation ${ }^{7}$.

Vertebrates also have CENP-Bs, which, like their $S$. pombe counterparts, bind to centromeres, and were only recently shown ${ }^{8}$ to contribute to centromere function. The vertebrate proteins also act on centromeric DNA repeats integrated on chromosomes outside the centromere. At these non-centromeric sites, the repeats become tightly packaged into transcriptionally inert protein-DNA complexes called heterochromatin in a way that is analogous to the action of CENP-Bs on the Tf 2 elements of S. pombe. It is not known whether CENP-Bs also regulate the activity of vertebrate transposable elements, or how the different roles of CENP-Bs in centromere formation and heterochromatin assembly are assigned. Nonetheless, this emerging story suggests a general role for CENP-Bs in helping the cell to manage its burden of repetitive DNA.

The packaging of transposable elements into transcriptionally inactive chromatin is not a defensive strategy limited to CENP-Bs. RNA interference also serves as a surveillance mechanism for double-stranded RNAs that result from the transcription of clustered or scrambled arrays of transposable elements ${ }^{9}$. This process leads to the degradation of messenger RNA transcripts of mobile elements, thus diminishing their activity. RNA interference also assembles mobile elements into heterochromatin, similar to the action of CENP-Bs on centromeric repeats and transposable elements.

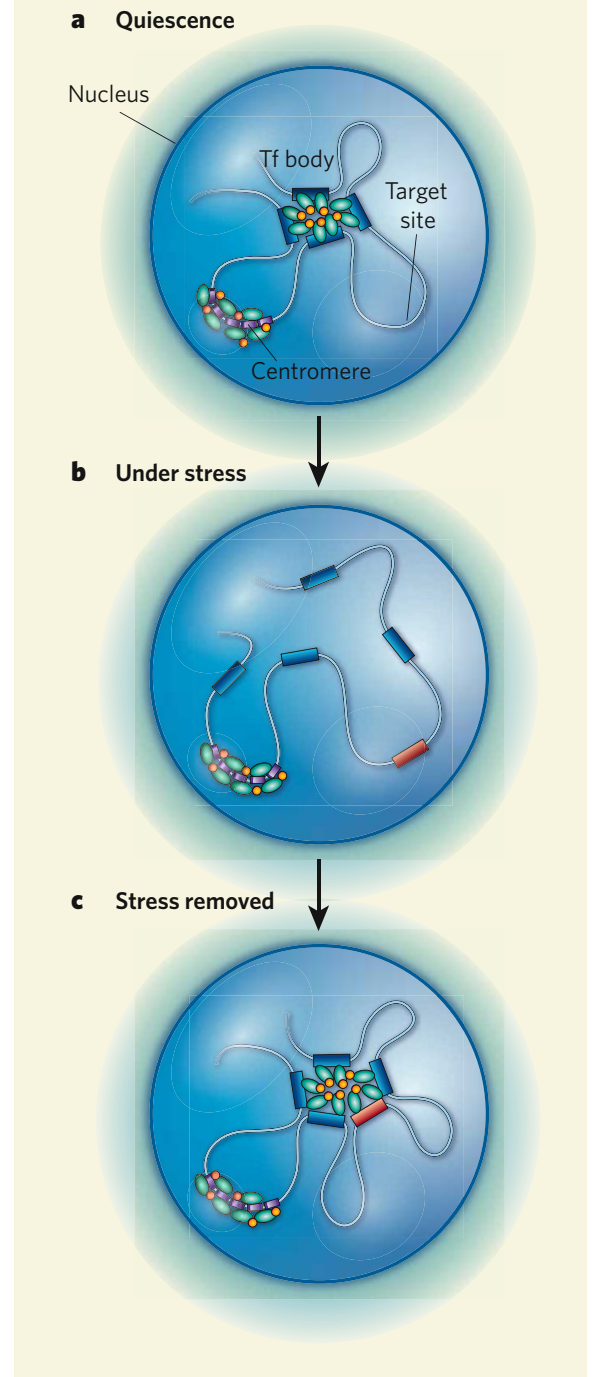

Figure 1 | Under the watchful eyes of CENP-B proteins. a, Cam et al. ${ }^{1}$ show that, as well as binding to centromeric DNA repeats (purple), CENP-Bs (green) bind to interspersed Tf elements (blue). CENP-Bs thus promote clustering of Tf elements into Tf bodies and recruit histone deacetylases (orange) that prevent the transcription of Tf elements. $\mathbf{b}$, In rare instances, such as exposure to oxidative stress, the Tf body disassembles, Tf-element expression is activated, and Tf elements can become mobile and transpose to new chromosomal locations (red). c, Once the stress subsides, newly transposed and preexisting Tf elements are packaged into Tf bodies.

Of course, the war between transposable elements and their host genome has been going on for millennia, allowing defensive strategies to assume other roles. The RNA-interference machinery in $S$. pombe, for example, has only a modest impact on Tf 2 expression $^{10}$, yet it is essential for the formation of heterochromatin at centromeric repeats, and is thus important for centromere function ${ }^{11}$. Also, a particularly remarkable finding of Cam et al. ${ }^{1}$ is that CENP-Bs regulate the expression of some genes adjacent to sites of Tf 2 insertion. This indicates that CENP-Bs and transposable elements collaborate to create genetic diversity, 
which could benefit the cell. The relationship between transposable elements and their host cell is made more complex by combative strategies that affect regular cellular functions. Undoubtedly, the future holds more surprises about the conflicts and collaborations between mobile elements and their host cell that contribute to genome function.

Daniel F. Voytas is in the Arnold and Mabel Beckman Center for Transposon Research, Department of Genetics, Cell Biology and Development, University of Minnesota, Minneapolis, Minnesota 55455, USA. e-mail: voytas@umn.edu

\title{
OPTICS
}

\section{Against the spread of the light}

\author{
Kishan Dholakia
}

\section{Light that propagates without spreading or diffracting sounds like a theorist's pipedream. But it is a very real proposition, and could be used to illuminate some profound aspects of wave-particle duality.}

The law of wave diffraction is a tough nut to crack. Consider, for example, a beam from a simple laser pointer. It might still seem narrow and pencil-like when you shine it on a wall or screen a few metres from you, but if you were to point it at the Moon, it would have a diameter of several hundred kilometres. But there are ways to beat the system, and one of them is investigated by Siviloglou et al. ${ }^{1}$, writing in Physical Review Letters. They have generated objects known as Airy wave packets that are entirely resistant to spreading or diffraction.

To appreciate the context of this advance, we must go back more than 100 years, to the time when it was becoming ever more apparent that classical mechanics and classical optics had significant shortcomings. Isaac Newton had originally proposed the idea that light came in discrete packets, which he called 'corpuscles'. But the wave-like behaviour of many optical phenomena swayed scientists towards the wave description of light.

With the advent of quantum mechanics, however, came the notion of wave-particle duality, which pops up in many different guises in physics. In this context, the concept of a wave packet takes on a pivotal, unifying significance. The wave packet is an 'envelope' containing an arbitrary number of waveforms, and also describes the probability that the particle or particles in a certain state will be measured to have a particular position and momentum. Owing to dispersion - waves of different frequencies travelling at different speeds - this wave packet should not remain unaltered. More generally, a given wave packet would then spread or diffract as it moves, changing its shape. wave-like behaviour of matter.
1. Cam, H. P., Noma, K., Ebina, H., Levin, H. L. \& Grewal, S. I. S. Nature 451, 431-436 (2008).

2. Earnshaw, W. C. et al. J. Cell Biol. 104, 817-829 (1987).

3. Irelan, J. T., Gutkin, G. I. \& Clarke, L. Genetics $157,1191-1203$ (2001).

4. McClintock, B. Science 226, 792-801 (1984).

5. Kipling, D. \& Warburton, P. E. Trends Genet. 13, 141-145 (1997).

6. Lin, R. et al. Science 318, 1302-1305 (2007).

7. Feschotte, C. \& Pritham, E. J. Annu. Rev. Genet. 41, 331-368 (2007).

8. Okada, T. et al. Cell 131, 1287-1300 (2007).

9. Slotkin, R. K. \& Martienssen, R. Nature Rev. Genet. 8, 272-285 (2007)

10. Hansen, K. R. et al. Mol. Cell. Biol. 25, 590-601 (2005).

11. Folco, H. D., Pidoux, A. L., Urano, T. \& Allshire, R. C. Science 319, 94-97 (2008) by Michael Berry and Nandor Balazs almost 30 years ago ${ }^{2}$. They are named after the nineteenth-century British astronomer George Biddell Airy, whose Airy function describes mathematically the way in which a star - an 'ideal' point source of light - is seen in a telescope. Owing to light's wave nature and the telescope's limited aperture, the ideal point image becomes a series of concentric ripples of bright and dark regions. In another demonstration of the underlying connections between matter and light, the Airy function is also the solution to Schrödinger's equation for a particle confined in a triangular-shaped potential well.

Siviloglou and colleagues ${ }^{1}$ shone a beam of visible light on a device known as a spatial light modulator. This is an array of liquid-crystal droplets that can present a varying path length to the wavefront of the incident light. Its effect is to mould the wavefront to a desired shape or function. The authors programmed it to produce an Airy function (Fig. 1) and looked at the beam's propagation characteristics. They confirmed that, as had been predicted ${ }^{2}$, the intensity profile of a beam of this shape remained unchanged ('propagation invariant') as the beam moved through free space.

They also verified other intriguing aspects of these beams, such as the ability of local intensity maxima in the Airy beam to be coaxed to perform ballistic dynamics, rather like a cannonball, and to follow a parabolic trajectory. Features within the beams were seen to 'accelerate' by starting to move transverse to their direction of propagation. This behaviour may mimic that of a quantummechanical particle placed in a constant gravitational field ${ }^{3}$. It would be interesting to see if one could generate such wave packets with matter waves, perhaps in a 'quantum degenerate gas' in which most atoms have settled into a ground state, creating a coherent matter wave.

In the absence of spreading or diffraction in light or matter, exciting applications become possible. Other light modes, such as Bessel beams ${ }^{4}$, have shown similar intriguing diffraction-free properties. These beams are now used to exert forces on minuscule particles (such as biological cells) in microfluidic environments, and to achieve targeted drug delivery into cells using ultrashort bursts of non-diffracting light ${ }^{5}$, with no precise focusing required. Stopping the spread of light or matter is a theorist's dream, but one with eminently practical consequences.

Kishan Dholakia is in the School of Physics and Astronomy, University of St Andrews, North Haugh, St Andrews KY16 9SS, UK. e-mail:kd1@st-andrews.ac.uk

ematical form of the Schrödinger equation (for particles) and the equation describing the diffraction of light. Light offers a very powerful way to explore such wave packets and their evolution, and to make telling analogies to the

The non-diffracting Airy wave packets generated by Siviloglou et al. ${ }^{1}$ were predicted
1. Siviloglou, G. A. Borky, J., Dogariu, A. \& Christodoulides, D. N. Phys. Rev. Lett. 99, 213901 (2007)

2. Berry, M. V. \& Balazs, N. L. Am. J. Phys. 47, 264-267 (1979).

3. Greenberger, D. M. Am. J. Phys. 48, 256 (1980).

4. Durnin, J., Miceli, J. J. Jr \& Eberly, J. H. Phys. Rev. Lett. 58, 1499-1501 (1986).

5. Tsampoula, X. et al. Appl. Phys. Lett. 91, 053902 (2007) 